\title{
A FATOU THEOREM FOR SOLUTIONS OF THE POROUS MEDIUM EQUATION
}

\author{
BJÖRN E. J. DAHLBERG, EUGENE B. FABES AND CARLOS E. KENIG ${ }^{1}$
}

\begin{abstract}
We show that nonnegative solutions of the porous medium equation in
\end{abstract} an infinite strip have nontangential initial values pointwise almost everywhere.

In this note we consider the existence of pointwise limits (Fatou type theorem) for each continuous nonnegative weak solution of the porous medium equation

$$
\partial u / \partial t=\Delta\left(u^{m}\right)
$$

in $S_{T} \equiv R^{n} \times(0, T]$ for some $T>0$. Here $m>1$ is a constant.

When $m=1, u$ is then a solution of the heat equation, and by the Widder representation theorem [W], there exists a unique nonnegative Borel measure $\mu$ such that

$$
\int_{R^{n}} e^{-|x|^{2} / 4 T} d \mu(x)<+\infty
$$

and

$$
u(y, t)=\frac{C_{n}}{t^{n / 2}} \int_{R^{n}} e^{-|x-y|^{2} / 4 t} d \mu(y) .
$$

Using standard facts about approximate identities (as in, for example [S, Chapter 3]), but with the parabolic dilations that are appropriate to the problem, one can show that there exists a set $Z \subset R^{n},|Z|=0$ (here $|E|$ stands for the Lebesgue measure of the set $E$ ), such that, for $x \in R^{n} \backslash Z$ and $\alpha>0$,

$$
\lim _{\substack{(y, t) \rightarrow x \\(y, t) \in \Gamma_{\alpha}(x)}} u(y, t)=f(x),
$$

where $f(x)=d \mu / d x$, the Radon-Nikodym derivative of $\mu$ with respect to Lebesgue measure, and $\Gamma_{\alpha}(x)$ is the parabolic nontangential approach region given by

$$
\Gamma_{\alpha}(x)=\{(y, t): 0<t<T,|y-x|<\alpha \sqrt{t}\} .
$$

Received by the editors August 12, 1983 and, in revised form, August 19, 1983

1980 Mathematics Subject Classification. Primary 35K65.

${ }^{1}$ The last two authors were supported in part by the N.S.F. The third author is an Alfred P. Sloan Fellow, 1981-1983. This research was started during a visit of the first author to the University of Minnesota under the auspices of the Ordway fund, and continued during a visit of the first and third authors to the Chalmers Institute of Technology in Goteborg, Sweden, under the auspices of the N.F.R. They would like to thank the University of Minnesota and the Chalmers Institute of Technology for their hospitality. 
The purpose of this work is to generalize the above result to any nonnegative continuous weak solution of (1). In doing so, the first obvious difficulty is that no representation formula of type (2) can be expected for solutions of (1), because of the nonlinear character of (1). Another difficulty we encounter is that equation (1) is not only nonlinear, but also degenerates at the points where $u=0$. In fact, there are solutions of (1), which for fixed $t$ have compact support in $x$ (for example, the Barrenblatt-Pattle function

$$
\begin{aligned}
& B(x, t)=t^{-n / k}\left\{\left(1-a r^{2} t^{-2 / k}\right)_{+}\right\}^{1 / m-1}, \\
& \quad r=|x|, k=n(m-1)+2, a=(m-1) / 2 m k) .
\end{aligned}
$$

One of our main tools (and the result that sparked our interest in a Fatou theorem for equation (1)) is the following theorem of Aronson and Cafarelli [AC]: every nonnegative continuous weak solution of (1) has a unique initial trace in the sense of measures, i.e. there exists a unique measure $\mu$ so that

$$
\lim _{t \rightarrow 0} \int_{R^{n}} u(x, t) \varphi(x) d x=\int_{R^{n}} \varphi(x) d \mu(x)
$$

for all $\varphi \in C_{0}\left(R^{n}\right)$. We also use repeatedly a Harnack type inequality of [AC] and the existence results of $[\mathbf{B C P}]$. Finally, we need a maximum principle and a uniqueness result which allow us to identify any solution with one of the solutions constructed in $[\mathbf{B C P}]$. These results were proved in $[\mathbf{D K}]$. With the aid of these theorems, we are able to reduce ourselves to a nondegenerate situation, to which an appropriate version of the linear theory (Lemma 2.2) applies.

We would like to thank Professor D. G. Aronson for making available to us the unpublished works [AC and BCP], as well as for interesting discussions.

The first section of the paper is devoted to notation and to recalling the results in [AC, BCP, and DK] needed in the sequel. $\S 2$ is devoted to the proof of our main result, Theorem 2.7.

1. Throughout this paper we will be dealing with continuous nonnegative weak solutions of (1) in $S_{T}=R^{n} \times(0, T], T>0$; i.e. with nonnegative functions $u$ continuous in $S_{T}$ satisfying

$$
\int_{R^{n}} \int_{\tau_{1}}^{\tau_{2}}\left(u^{m} \Delta \varphi+u \frac{\partial \varphi}{\partial t}\right) d x d t=\left.\int_{R^{n}} u \varphi\right|_{t=\tau_{2}} d x-\left.\int_{R^{n}} u \varphi\right|_{t=\tau_{1}} d x
$$

for all $\tau_{i}$, such that $0<\tau_{1}<\tau_{2} \leqslant T$, and all $\varphi \in C^{2,1}\left(S_{T}\right)$, such that $\varphi(-, t)$ has compact support for all $t \in\left[\tau_{1}, \tau_{2}\right] . B_{r}=B_{r}(0)$ will denote the ball in $x$ with center at 0 and radius $r$. If $\nu$ is a nonnegative Borel measure, $k=n(m-1)+2$,

$$
\|\mid \nu\|=\sup _{r \geqslant 1} \frac{1}{r^{k / m-1}} \int_{B_{r}} d \nu .
$$

THEOREM $1.1[\mathbf{A}, \mathbf{C}]$. Let $u$ be a continuous weak solution of (1) in $S_{T}$. There exists a unique nonnegative Borel measure $\mu$ on $R^{n}$ such that

$$
\lim _{t \downarrow 0^{+}} \int_{R^{n}} \varphi(x) u(x, t) d x=\int_{R^{n}} \varphi(x) d \mu(x)
$$


for all $\varphi \in C_{0}\left(R^{n}\right)$. Moreover, there exists a constant $C=C(n, m)$ such that

$$
\int_{B_{r}} d \mu \leqslant C\left\{r^{k /(m-1)} T^{-1 / m-1}+T^{n / 2} u^{k / 2}(0, T)\right\} .
$$

For a measure $\nu,\|\nu\|<+\infty$, define

$$
l(\nu)=\lim _{r \rightarrow \infty} \sup _{R \geqslant r} \frac{1}{R^{k / m-1}} \int_{B_{R}} d \nu .
$$

THEOREM 1.2 [BCP]. There exist constants $c_{1}, c_{2}, c_{3}$ depending only on $n, m$ such that, given any Borel measure $\nu$ with $\|\nu\|<+\infty$, there exists a function $v(x, t)$ defined for $0<t<T(\nu)=c_{1} / l(\nu)^{m-1}$ such that:

(a) $v(x, t)^{(m-1)} \leqslant c_{2} t^{-n(m-1) / k}\left(1+|x|^{2}\right)\|\nu\|^{2(m-1) / k}$, for $0<t<T_{0}(\nu)=$ $c_{1} /\|\nu\|^{m-1}$

(b) $t \rightarrow v(-, t) \in C\left[(0, T(\nu)) ; L_{\mathrm{loc}}^{1}\left(R^{n}\right)\right]$;

(c) $\|v(-, t)\| \leqslant c_{3}\|v\|$ for $0<t<T_{0}(\nu)$;

(d) for $\psi \in C_{0}^{\infty}\left(R^{n} \times[0, T(\nu))\right)$,

$$
\int_{0}^{T(\nu)} \int_{R^{n}}\left(v^{m} \Delta \psi+v \frac{\partial \psi}{\partial t}\right) d x d t=-\int_{R^{n}} \psi(x, 0) d \nu(x)
$$

REMARK 1.3. P. Sacks has shown that $v$ is continuous in $S_{T(\nu)}$. It then follows that $v$ is a continuous weak solution of (1) by a simple approximation argument. Because of estimates (c) and (a), $v^{m}$ is in $L^{1}\left(K \times\left[0, T_{0}(\nu)\right]\right)$ for any $K$ compact in $R^{n}$. As a consequence of this, the fact that $v$ is a continuous weak solution of (1) and (d), it is easy to see that for $\psi \in C_{0}^{\infty}\left(R^{n}\right)$,

$$
\lim _{\tau_{1} \rightarrow 0} \int_{R^{n}} v\left(x, \tau_{1}\right) \psi(x) d x=\int_{R^{n}} \psi(x) d \nu(x) .
$$

Because of Theorem 1.1, we see then that $v(-, t) \rightarrow \nu$ weakly in the sense of measures as $t \rightarrow 0$.

THEOREM $1.4[\mathrm{DK}]$. Let $u(x, t), v(x, t)$ be two nonnegative, continuous weak solutions of (1) in $S_{T}$. Let $\mu$ and $\nu$, respectively, be the nonnegative Borel measures associated to them as in Theorem 1.1. If $\mu \leqslant \nu$ (in the sense of Borel measures), then $u(x, t) \leqslant v(x, t)$ for all $(x, t) \in S_{T}$.

REMARK. As a consequence of Theorem 1.4, the estimates in Theorem 1.2 apply to any continuous weak solution of (1).

The last notation we introduce is the following: for $x \in R^{n}, \alpha>0, \Gamma_{\alpha}\left(x_{0}\right)=$ $\left\{(x, t): t>0,\left|x-x_{0}\right|<\alpha \sqrt{t}\right\}$. If $h>0$,

$$
\Gamma_{\alpha}^{h}\left(x_{0}\right)=\left\{(x, t):(x, t) \in \Gamma_{\alpha}\left(x_{0}\right), 0<t<h\right\} .
$$

2. After stating and proving some preliminary lemmas, we establish our main result, Theorem 2.7 .

LEMMA 2.1. Let $u \geqslant 0$ be a nonnegative, continuous weak solution of (1) in $S_{T}$. Let $K \subset R^{n}$ be compact. Then, given $\varepsilon>0, \alpha>0, h<T$, there exist $F \subset K, F$ closed with $|K \backslash F|<\varepsilon$, and a number $M=M(\alpha, h, \varepsilon, u, K)$ such that $u(x, t) \leqslant M$ for all $(x, t)$ $\in R=\cup_{x_{0} \in F} \Gamma_{\alpha}^{h}\left(x_{0}\right)$. 
Proof. Let $\mu$ be the Borel measure associated to $u$ as in Theorem 1.1. As is well known, given $\varepsilon>0$, there exist a subset $F \subset K$ and a number $N$ so that for each $x_{0} \in F$,

$$
\sup _{0<r<1} \frac{1}{\left|B\left(x_{0}, r\right)\right|} \int_{B\left(x_{0}, r\right)} d \mu(x) \leqslant N,
$$

and such that $|K \backslash F|<\varepsilon$. Now fix $x_{0} \in F, t_{0}<h$ and $\alpha$. We can obviously assume $t_{0}$ is sufficiently small and $\alpha$ is sufficiently large. Now consider the function

$$
v(x, t)=\frac{1}{\alpha^{2 / m-1}} u\left(\alpha \sqrt{t_{0}}\left(x+x_{0}\right), t_{0} t\right),
$$

which is a continuous weak solution of (1) in the strip $S_{T / t_{0}}$. Let $\nu$ be its weak limit. Clearly, $\nu$ is the measure which, to a Borel set $E$, assigns the mass

$$
\frac{1}{\alpha^{2 / m-1}} \frac{1}{\left(\alpha \sqrt{t_{0}}\right)^{n}} \mu(\tilde{E})
$$

where $\tilde{E}$ is the set $E-x_{0}$ dilated by a factor of $\alpha \sqrt{t_{0}}$. We first proceed to estimate $\|\nu\|$. Clearly,

$$
\frac{1}{R^{k / m-1}} \int_{B(0, R)} d \nu=\frac{1}{(\alpha R)^{2 / m-1}} \frac{1}{\left(\alpha \sqrt{t_{0}} R\right)^{n}} \int_{B\left(x_{0}, \alpha \sqrt{t_{0}} R\right)} d \mu,
$$

and we are interested in $R>1$. Consider the first case when $1 \leqslant R \leqslant 1 / \alpha \sqrt{t_{0}}$. Then the right-hand expression is bounded (by our choice of $x_{0}$ ) by $N / \alpha^{2 / n-1}$ which, choosing $\alpha^{2} \geqslant N^{m-1} / c_{1}$, is less than or equal to $c_{1}^{1 / m-1}$ (here $c_{1}$ is the constant in Theorem 1.2). Next, assume $R \alpha \sqrt{t_{0}} \geqslant 1$. Then the right-hand expression above is bounded by

$$
t_{0}^{1 / m-1} \frac{1}{\left(\alpha \sqrt{t_{0}} R\right)^{2 / m-1}} \frac{1}{\left(\alpha \sqrt{t_{0}} R\right)^{n}} \int_{B\left(x_{0}, \alpha \sqrt{t_{0}} R\right)} d \mu
$$

Let $\lambda=\alpha \sqrt{t_{0}} R$ and use the estimate in Theorem 1.1 applied to $u\left(x-x_{0}, t\right)$. Since $\lambda \geqslant 1$, we obtain a bound of

$$
C t_{0}^{1 / m-1}\left\{T^{-1 / m-1}+T^{n / 2} u^{k / 2}\left(x_{0}, T\right)\right\} .
$$

This is less than or equal to $c_{1}^{1 / m-1}$ by choosing $t_{0}$ sufficiently small depending on $T, u$ and $K$. We have then shown, with our choice of $\alpha, t_{0}$, that $\|\nu\|<c_{1}^{1 / m-1}$. We can also choose $t_{0}$ so small that $1 \leqslant T / t_{0}$. We then see (using Theorems 1.4 and 1.2(a)) that $\sup _{|x| \leqslant 1} v(x, 1) \leqslant C$ for some absolute constant $C$. But

$$
\sup _{|x| \leqslant 1} v(x, 1)=\frac{1}{\alpha^{2 / m-1}} \sup _{\left|x-x_{0}\right|<\alpha \sqrt{t_{0}}} u\left(x, t_{0}\right)
$$

and the lemma follows.

The next result is the version of the linear theory that we will need. To maintain the flow of the proof, we postpone its proof until the end of the paper. 
LEMMA 2.2. Let $0 \leqslant v \leqslant M$ be a solution in $R=\cup_{x_{0} \in F} \Gamma_{\alpha}^{h}\left(x_{0}\right)$ of a linear equation of the form $\partial v / \partial t-\operatorname{div}\left(a_{i j}(x, t) \nabla v\right)=0$, where $\left(a_{i j}(x, t)\right)$ is an elliptic matrix, i.e.

$$
\lambda|\xi|^{2} \leqslant \sum a_{i j}(x, t) \xi_{i} \xi_{j} \leqslant \Lambda|\xi|^{2} .
$$

Then for almost every $x_{0} \in F, \lim _{(x, t) \rightarrow x_{0} ;(x, t) \in R} v(x, t)$ exists and is finite.

We now continue our development.

LEMMA 2.3. Let $0 \leqslant u$ be a continuous weak solution of (1) in $S_{T}$. Let

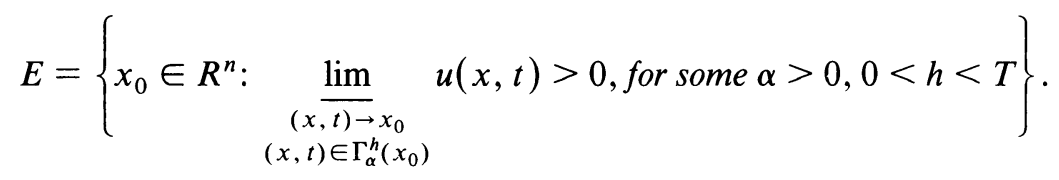

Then for almost every $x_{0} \in E, \lim _{(x, t) \rightarrow x_{0} ;(x, t) \in \Gamma_{\beta}^{k}\left(x_{0}\right)} u(x, t)$ exists and is finite for all $\beta>0, k<T$.

Proof. By a familiar point of density argument (see [C]), given $\alpha, \beta>0, h>0$, $\varepsilon>0$ and a closed set $F$, there exists a closed set $F_{0} \subset F,\left|F \backslash F_{0}\right|<\varepsilon$ and $k>0$ so that $\cup_{x_{0} \in F_{0}} \Gamma_{\beta}^{k}\left(x_{0}\right) \subset \cup_{x_{0} \in F} \Gamma_{\alpha}^{h}\left(x_{0}\right)$. Therefore, it is easy to see, using Lemma 2.1, that it is enough to show that if on $R=\cup_{x_{0} \in F} \Gamma_{\alpha}^{h}\left(x_{0}\right)$ we have $\varepsilon \leqslant u(x, t) \leqslant 1 / \varepsilon$, then $\lim _{(x, t) \rightarrow x_{0} ;(x, t) \in R} u(x, t)$ exists and is finite for a.e. $x_{0} \in F$. It is not hard to see that in such an $R, \nabla u \in L_{\text {loc }}^{2}($ see $[\mathbf{D K}])$ and $\Delta u^{m}=\operatorname{div}\left(m u^{m-1} \nabla u\right)=\partial u / \partial t$ in the weak sense. Hence, Lemma 2.3 follows from 2.2, taking $\left(a_{i j}(x, t)\right)=$ $\left(m u^{m-1}(x, t) \delta_{i j}\right)$.

LEMMA 2.4. Let $u$ be a nonnegative, continuous weak solution of (1) in $S_{T}$. Let $\mu$ be the measure associated to it in Theorem 1.1 and $f=d \mu / d x$, the Radon-Nikodym derivative of $\mu$ with respect to Lebesgue measure. Let $E=\left\{x_{0} \in R^{n}: f\left(x_{0}\right)=0\right\}$. Then for almost every $x_{0} \in E$ we have

$$
\lim _{\substack{(x, t) \rightarrow x_{0} \\(x, t) \in \Gamma_{\alpha}^{h}\left(x_{0}\right)}} u(x, t)=0 \quad \text { for } \alpha>0, h<T .
$$

Proof. Let $d \mu_{\varepsilon}=d \mu+\varepsilon d x$. For $0<\varepsilon<\frac{1}{2},\left\|\mu_{\varepsilon}\right\| \leqslant C$ where $C$ depends only on $\mu$. Use Theorem 1.2 to solve the initial value problem $\partial u_{\varepsilon} / \partial t-\Delta\left(u_{\varepsilon}\right)^{m}=0$ in $R^{n} \times(0, T], u_{\varepsilon}(-, t) \rightarrow t \rightarrow 0 d \mu_{\varepsilon}$ weakly, where $T$ depends only on $\mu$. By Theorem 1.4 we have, for $0<t \leqslant T, u_{\varepsilon}(x, t) \geqslant u(x, t)$ and $u_{\varepsilon}(x, t) \geqslant \varepsilon$. By Lemma 2.3 $\lim _{(x, t) \rightarrow x_{0} ;(x, t) \in \Gamma_{\alpha}^{h}\left(x_{0}\right)} u_{\varepsilon}(x, t)$ exists for a.e. $x_{0} \in R^{n}$. Let $h(x)=\lim _{t \rightarrow 0} u_{\varepsilon}(x, t)$ and let $\theta \in C_{0}\left(R^{n}\right), 0 \leqslant \theta$. Then by Fatou's lemma

$$
\int_{R^{n}} h \cdot \theta d x \leqslant \int_{R^{n}} \theta(x)[\varepsilon d x+d \mu(x)] .
$$

Choosing for $\theta$ an approximate identity, we see that $h(x) \leqslant \varepsilon+f(x)$ a.e. Thus, $h(x) \leqslant \varepsilon$ for a.e. $x \in E$. But

$$
\lim _{\substack{(x, t) \rightarrow x_{0} \\(x, t) \in \Gamma_{\alpha}^{h}\left(x_{0}\right)}} u(x, t) \leqslant \lim _{\substack{(x, t) \rightarrow x_{0} \\(x, t) \in \Gamma_{\alpha}^{h}\left(x_{0}\right)}} u_{\varepsilon}(x, t) \leqslant \varepsilon \text { for a.e. } x_{0} \in E,
$$

and the lemma follows. 
LEMMA 2.5. Let $u$ be a nonnegative, continuous weak solution of (1) in $S_{T}$. Let $\mu$ be the measure associated to it in Theorem 1.1, and $f=d \mu / d x$, the Radon-Nikodym derivative of $\mu$ with respect io Lebesgue measure. Let $E=\left\{x \in R^{n}: f(x)>0\right\}$. Then for almost every $x_{0} \in E$,

$$
\underset{\substack{(x, t) \rightarrow x_{0} \\(x, t) \in \Gamma_{\alpha}^{h}\left(x_{0}\right)}}{\lim } u(x, t)>0 \text { for some } \alpha, h .
$$

Proof. For a.e. $x_{0} \in R^{n}$,

$$
\lim _{\substack{|B| \rightarrow 0 \\ x_{0} \in B}} \frac{1}{|B|} \int_{B} d \mu(y)=f\left(x_{0}\right)
$$

where $B$ is a ball. Fix one such $x_{0}$ in $E$, with $f\left(x_{0}\right)=2 \varepsilon$. We can then assume $(1 /|B|) \int_{B} d \mu(y) \geqslant \varepsilon$ for all balls containing $x_{0}$ of radius $r \leqslant r_{0}$. We now use the estimate in Theorem 1.1. Let $\left|x-x_{0}\right|<\alpha \sqrt{t}, r=\alpha \sqrt{t}$, and choose $T=t$ in 1.1. Then

$$
\int_{B(x, \alpha \sqrt{t})} d \mu(y) \leqslant C\left\{(\alpha \sqrt{t})^{k / m-1}+t^{n / 2} u^{k / 2}(x, t)\right\},
$$

so

$$
\int_{B(x, \alpha \sqrt{t})} d \mu(y) \leqslant C\left\{(\alpha \sqrt{t})^{n}(\alpha \sqrt{t})^{2 / m-1} t^{-1 / m-1}+(\sqrt{t})^{n} u^{k / 2}(x, t)\right\} .
$$

Dividing by $|B(x, \alpha \sqrt{t})|$, we see that

$$
\frac{1}{|B(x, \alpha \sqrt{t})|} \int_{B(x, \alpha \sqrt{t})} d \mu(y) \leqslant C\left\{\alpha^{2 / m-1}+\alpha^{-n} u^{k / 2}(x, t)\right\} .
$$

If $\alpha \sqrt{t}<r_{0}$, the left-hand side exceeds $\varepsilon$, so $\varepsilon \leqslant C\left\{\alpha^{2 / m-1}+\alpha^{-n} u^{k}(x, t)\right\}$. If we then first choose $\alpha$ so that $C \alpha^{2 / m-1} \leqslant \varepsilon / 2$, and choose $t$ so small that $\alpha \sqrt{t}<r_{0}$, then $u^{k}(x, t) \geqslant C^{-1} \alpha^{n} \varepsilon / 2$, and the lemma follows.

LEMMA 2.6. Let $u$ be a nonnegative, continuous weak solution of (1) in $S_{T}$. Then, for a.e. $x_{0} \in R^{n}, \lim _{(x, t) \rightarrow x_{0}} ;(x, t) \in \Gamma_{\beta}^{k}\left(x_{0}\right) u(x, t)$ exists and is finite for all $\beta>0, k<T$. Moreover, if $g\left(x_{0}\right)=\lim _{(x, t) \rightarrow x_{0} ;(x, t) \in \Gamma_{\beta}^{k}\left(x_{0}\right)} u(x, t)$, we have $g\left(x_{0}\right) \leqslant f\left(x_{0}\right)$ for almost every $x_{0}$, where $f(x)=d \mu / d x$ and $\mu$ is the measure associated to $u$ in Theorem 1.1.

Proof. The first part of the lemma follows directly from Lemmas 2.5, 2.3 and 2.4. The second part follows by Fatou's lemma, as in the last part of the proof of Lemma 2.4 .

We are now ready to state and prove the main theorem in the paper.

THEOREM 2.7. Let $u$ be a nonnegative, continuous weak solution of (1) in $S_{T}$. Then, for almost every $x_{0} \in R^{n}, \lim _{(x, t) \rightarrow x_{0} ;(x, t) \in \Gamma_{\beta}^{k}\left(x_{0}\right)} u(x, t)$ exists and is finite for all $\beta>0, k<T$. Moreover, if $\mu$ is the measure associated to $u$ in Theorem 1.1, and $f(x)=d \mu / d x$ is the Radon-Nikodym derivative of $\mu$ with respect to Lebesgue measure, then for almost every $x_{0} \in R^{n}$,

$$
\lim _{\substack{(x, t) \rightarrow x_{0} \\(x, t) \in \Gamma_{\beta}^{k}\left(x_{0}\right)}} u(x, t)=f\left(x_{0}\right) .
$$


Proof. Because of Lemma 2.6, if

$$
g\left(x_{0}\right)=\lim _{\substack{(x, t) \rightarrow x_{0} \\(x, t) \in \Gamma_{\beta}^{k}\left(x_{0}\right)}} u(x, t)
$$

all we have to show is that $g\left(x_{0}\right) \geqslant f\left(x_{0}\right)$ for almost every $x_{0} \in R^{n}$. To show this, let $f_{N}(x)=\max \{f(x), N\}$. Use Theorem 1.2 to solve the initial value problem

$$
\frac{\partial u_{N}}{\partial t}=\Delta u_{N}^{m} \quad \text { in } R^{n} \times(0, \bar{T}], \quad u_{N}(-, t) \underset{t \rightarrow 0}{\rightarrow} f_{N}(x) d x
$$

weakly, where $\bar{T}$ depends only on $\mu$. By Theorem $1.4, u_{N}(x, t) \leqslant N, u_{N}(x, t) \leqslant$ $u(x, t)$ for $0<t \leqslant \bar{T}$. Let $g_{N}\left(x_{0}\right)=\lim _{t \rightarrow 0} u_{N}\left(x_{0}, t\right)$, which exists for almost every $x_{0}$ by Lemma 2.6. By dominated convergence (since $u_{N}(x, t)<N$ ) we see that $g_{N}\left(x_{0}\right)=f_{N}\left(x_{0}\right)$ for a.e. $x_{0}$. Also, as $u_{N}(x, t) \leqslant u(x, t), g_{N}\left(x_{0}\right) \leqslant g\left(x_{0}\right)$. The theorem now follows by letting $N \rightarrow \infty$.

We now turn to the proof of Lemma 2.2. The proof is a simple adaptation of the argument in [C].

Proof of Lemma 2.2. We will be working in the strip $S_{T}=R^{n} \times(0, T]$, and also considering the domains $R_{j}=R \cap\{(x, t): t>1 / j\}$ and $S_{j}=R^{n} \times(1 / j, T]$.

By a well-known theorem of Aronson [A], the fundamental solution $g(x, t, \xi, T)$ of $\partial v / \partial t-\operatorname{div}\left(a_{i j}(x, t) \nabla v\right)=0$ in $S_{T}$ satisfies the estimates

$$
K^{-1} \gamma_{1}(x-\xi, t-\tau) \leqslant g(x, t, \xi, T) \leqslant K \gamma_{2}(x-\xi, t-\tau),
$$

for all $(x, t),(\xi, \tau) \in S_{T}$, with $t>\tau$, where $\gamma_{i}(x, t)$ is the fundamental solution of $\partial v / \partial t-\alpha_{i} \Delta v=0$, where $\alpha_{i}$ are constants depending only on the ellipticity constants of $\left(a_{i j}(x, t)\right)$. Consider first the case when $0 \leqslant u \leqslant M$ is a solution of $\partial u / \partial t-\operatorname{div}\left(a_{i j}(x, t) \nabla u\right)=0$ in all $S_{T}$. By another theorem of Aronson [ $\left.\mathbf{A}_{2}\right]$, we have the Widder type representation formula

$$
u(x, t)=\int_{R^{n}} g(x, t, \xi, 0) f(\xi) d \xi, \quad \text { where } f \in L^{\infty}\left(R^{n}\right) .
$$

Since

$$
\int g(x, t, \xi, 0) h(\xi) d \xi \underset{\substack{(x, t) \rightarrow x_{0} \\(x, t) \in S_{T}}}{\rightarrow} h\left(x_{0}\right), \quad \text { whenever } h \in C_{0}\left(R^{n}\right),
$$

the result follows in this case by the upper bound on $g(x, t, \xi, 0)$ and well-known techniques (see [S]). Let us return now to the general case and consider the functions $\varphi_{j}(x)=v(x, 1 / j) \chi_{R_{j}}(x, 1 / j)$, and let

$$
u_{j}(x, t)=\int_{R^{n}} g_{j}(x, t, \xi, 1 / j) \varphi_{j}(\xi) d \xi,
$$

where $g_{j}(x, t, \xi, \tau)$ is the fundamental solution of $\partial v / \partial t-\operatorname{div}\left(a_{i j}(x, t) \nabla v\right)=0$ on $S_{j}$. The $u_{j}(x, t)$ are bounded solutions in $S_{j}$. For $(x, t) \in R_{j}$, define $w_{j}(x, t)$ by $v(x, t)=u_{j}(x, t)+w_{j}(x, t)$. Clearly, $w_{j}$ is a solution in $R_{j}$. Since the $u_{j}$ are uniformly bounded, a subsequence, which we still denote by $u_{j}$, converges uniformly on compact subsets of $S_{T}$ to a nonnegative bounded solution $u$ in $S_{T}$. Hence, $\left\{w_{j}(x, t)\right\}$ also converges uniformly on compact subsets of $R$ to a bounded solution 
$w$. Clearly, $v(x, t)=u(x, t)+w(x, t)$ in $R$. Since $u$ has nontangential limits a.e., it is enough to show that $w(x, t) \rightarrow 0$ as $(x, t) \rightarrow x_{0} \in F,(x, t) \in R$, for a.e. $x_{0} \in F$. Consider now $h(x, t)=\int_{c_{F}} g(x, t, \xi, 0) d \xi$. This is a nonnegative, bounded solution on $S_{T}$. The lower bound for $g$, together with a rescaling argument, show there exists a constant $C$ such that $h(x, t) \geqslant C$ on $\partial R \backslash F$. Hence, by the maximum principle on $R_{j}, w_{j}(x, t) \leqslant(2 M / C) h(x, t)$ on $R_{j}$, and hence $w(x, t) \leqslant(2 M / C) h(x, t)$ on $R$. But then, as $h(x, t) \rightarrow 0$ nontangentially almost everywhere on $F$, the proof is complete.

\section{REFERENCES}

[A] D. G. Aronson, Bounds for the fundamental solution of a parabolic equation, Bull. Amer. Math. Soc. 73 (1967), 890-897.

$\left[\mathbf{A}_{2}\right] \ldots$, Non-negative solutions of linear parabolic equations: an addendum, Ann. Scuola Norm. Sup. Pisa Cl. Sci. (4) 24 (1971), 221-228.

[AC] D. G. Aronson and L. A. Caffarelli, The initial trace of a solution of the porous medium equation, preprint.

[BCP] P. Benilan, M. Crandall and M. Pierre, Solutions of the porous media equation in $R^{N}$, under optimal conditions on initial values, Indiana Univ. Math. J. (to appear).

[C] A. P. Calderon, On the behavior of harmonic functions at the boundary, Trans. Amer. Math. Soc. 68 (1950), 47-54.

[DK] B. E. J. Dahlberg and C. E. Kenig, Non-negative solutions of the porous medium equation, in preparation.

[S] E. M. Stein, Singular integrals and differentiability properties of functions, Princeton Univ. Press, Princeton, N. J., 1971.

[W] D. Widder, Positive temperature on the infinite rod, Trans. Amer. Math. Soc. 55 (1944), 85-95.

School of Mathematics, University of Minnesota, Minneapolis, Minnesota 55455 (Current address of E. B. Fabes and C. E. Kenig)

Department of Mathematics, Uppsala University, UpPSala, Sweden (Current address of Björn E. J. Dahlberg) 